\title{
A solvable model for the diffusion and reaction of neurotransmitters in a synaptic junction
}

\author{
Jorge L Barreda, Huan-Xiang Zhou*
}

\begin{abstract}
Background: The diffusion and reaction of the transmitter acetylcholine in neuromuscular junctions and the diffusion and binding of $\mathrm{Ca}^{2+}$ in the dyadic clefts of ventricular myocytes have been extensively modeled by Monte Carlo simulations and by finite-difference and finite-element solutions. However, an analytical solution that can serve as a benchmark for testing these numerical methods has been lacking.

Result: Here we present an analytical solution to a model for the diffusion and reaction of acetylcholine in a neuromuscular junction and for the diffusion and binding of $\mathrm{Ca}^{2+}$ in a dyadic cleft. Our model is similar to those previously solved numerically and our results are also qualitatively similar.

Conclusion: The analytical solution provides a unique benchmark for testing numerical methods and potentially provides a new avenue for modeling biochemical transport.
\end{abstract}

\section{Background}

In intercellular and intracellular spaces, passive transport of biomolecules is a common phenomenon. Because such processes are difficult to probe directly by experiments, numerical modeling is increasingly used to gain insight. Two processes that have been extensively modeled are the diffusion and reaction of the transmitter acetylcholine in a neuromuscular junction [1-6] and the diffusion and binding of $\mathrm{Ca}^{2+}$ in the dyadic cleft of a ventricular myocyte $[7,8]$. In contrast to previous numerical approaches, here we present an analytical solution of a model for the diffusion and reaction of acetylcholine in a synaptic cleft (or $\mathrm{Ca}^{2+}$ in a dyadic cleft). Our model is similar to those previously solved numerically; hence our analytical solution potentially provides a new avenue for modeling biochemical transport. More importantly, an analytical solution provides a unique benchmark for testing numerical methods. Such a solution has been lacking up to now; the present work fills this gap.

Neuromuscular junction refers to the cleft between a motor neuron and a muscle fiber. As illustrated in Figure 1, the neuronal signal for muscle contraction is mediated by acetylcholine. These neurotransmitter molecules are initially inside vesicles located in the pre-

\footnotetext{
* Correspondence: hzhou4@fsu.edu
Department of Physics and Institute of Molecular Biophysics, Tallahassee,

* Correspondence: hzhou4@fsu.edu Florida 32306, USA
} (10ida 32306, USA

\section{( Biomed Central}

(c) 2011 Barreda and Zhou; licensee BioMed Central Ltd. This is an Open Access article distributed under the terms of the Creative Commons Attribution License (http://creativecommons.org/licenses/by/2.0), which permits unrestricted use, distribution, and reproduction in any medium, provided the original work is properly cited.

synaptic axon terminal. When an action potential reaches the axon terminal, the vesicles release acetylcholine molecules into the synaptic cleft. These molecules then diffuse toward the post-synaptic membrane and bind to acetylcholine receptors in the membrane. Acetylcholine binding activates these ligand-gated ion channels, allowing $\mathrm{Na}^{+}$to flow in and generating an action potential along the muscle fiber. Finally excess acetylcholine molecules around the post-synaptic membrane are broken down by acetylcholinesterase to prevent continued activation of acetylcholine receptors.

A related system is a dyadic cleft, which spans the gap between the cell membrane in a transverse tubule and the membrane of a sarcoplasmic reticulum. As Figure 2 illustrates, $\mathrm{Ca}^{2+}$ can enter the cell through L-type $\mathrm{Ca}^{2+}$ channels on the cell membrane in response to the arrival of an action potential. The ions then diffuse to reach and activate ryaonodine receptors in the membrane of the sarcoplasmic reticulum. The activated ryaonodine receptors release $\mathrm{Ca}^{2+}$ from the sarcoplasmic reticulum, which ultimately lead to muscle contraction.

Here we propose a simple but not unrealistic model for the diffusion and reaction of acetylcholine in a neuromuscular junction. The model also applies to the diffusion and binding of $\mathrm{Ca}^{2+}$ in a dyadic cleft. We are able to find an analytical solution for this model. For convenience we describe our model in the language of neuromuscular 


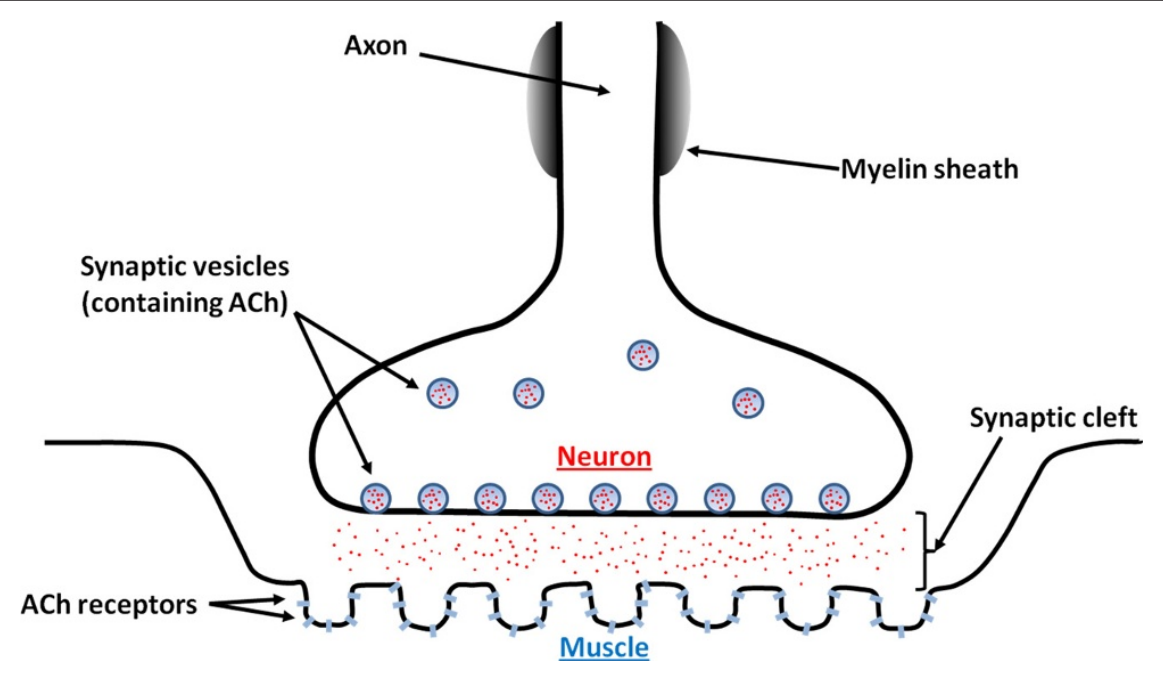

Figure 1 Illustration of a neuromuscular junction. Presented are the key players in the diffusion and reaction of the neurotransmitter, acetylcholine (ACh).

junction. As shown in Figure 3, we model the synaptic cleft as the space between two infinite, parallel planar membranes. In the pre-synaptic membrane, there is a periodic array of circular openings, via which acetylcholine molecules enter the cleft. In the post-synaptic membrane, there is a periodic array of circular disks, where the acetylcholine molecules are absorbed. The quantity of interest is the total flux, $J(t)$, at time $t$ of acetylcholine molecules across the post-synaptic membrane. This model allows us to use periodic boundary conditions in the transverse direction. Our results are qualitatively similar to those obtained previously by numerical solutions [1-7]. More realistic ingredients can be added to our model and still permit analytical solutions.

\section{Methods}

We set up a coordinate system such that the $x$ and $y$ axes are parallel to the pre- and post-synaptic membranes. The synaptic junction has depth $L_{z}$. The junction is periodic in the $x$ and $y$ directions, with periodicities of $L_{x}$ and $L_{y}$, respectively. In each "unit cell", a synaptic vesicle bursts at time $t=0$, releasing

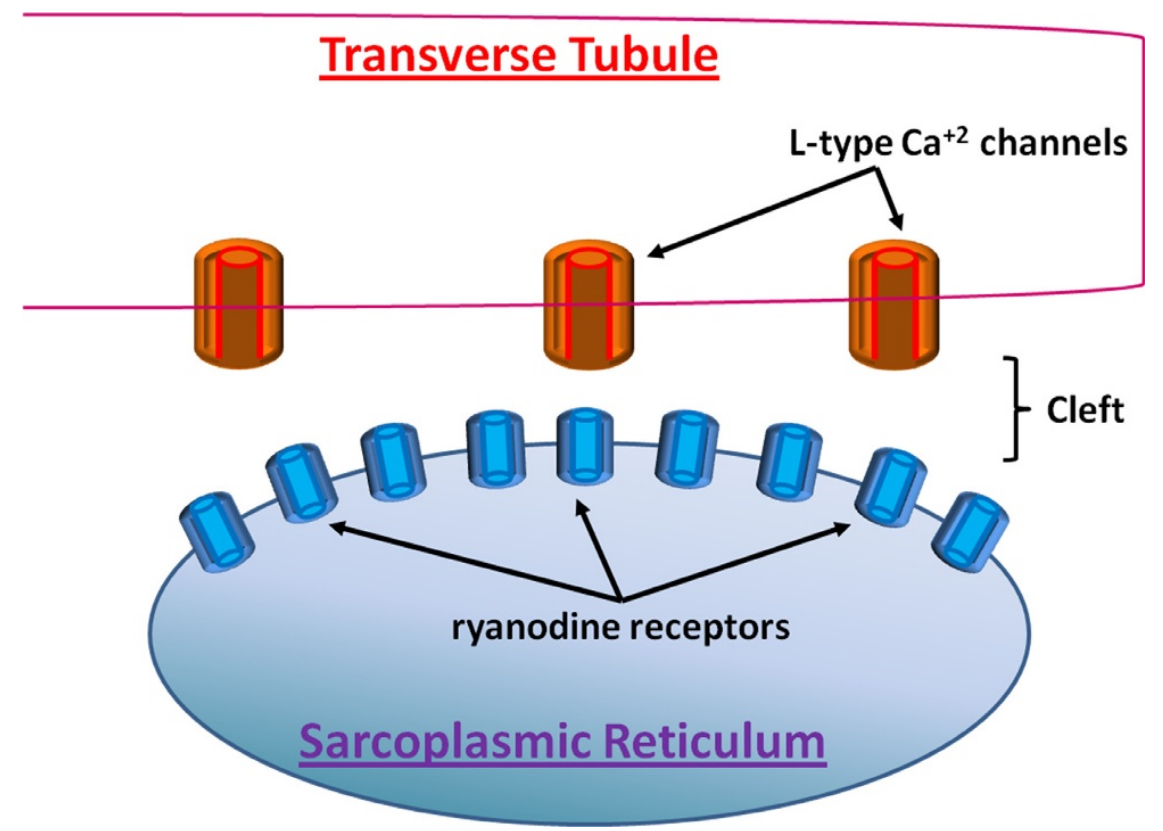

Figure 2 Illustration of a dyadic cleft. Presented are the key players in the diffusion and binding of $\mathrm{Ca}^{2+}$ 

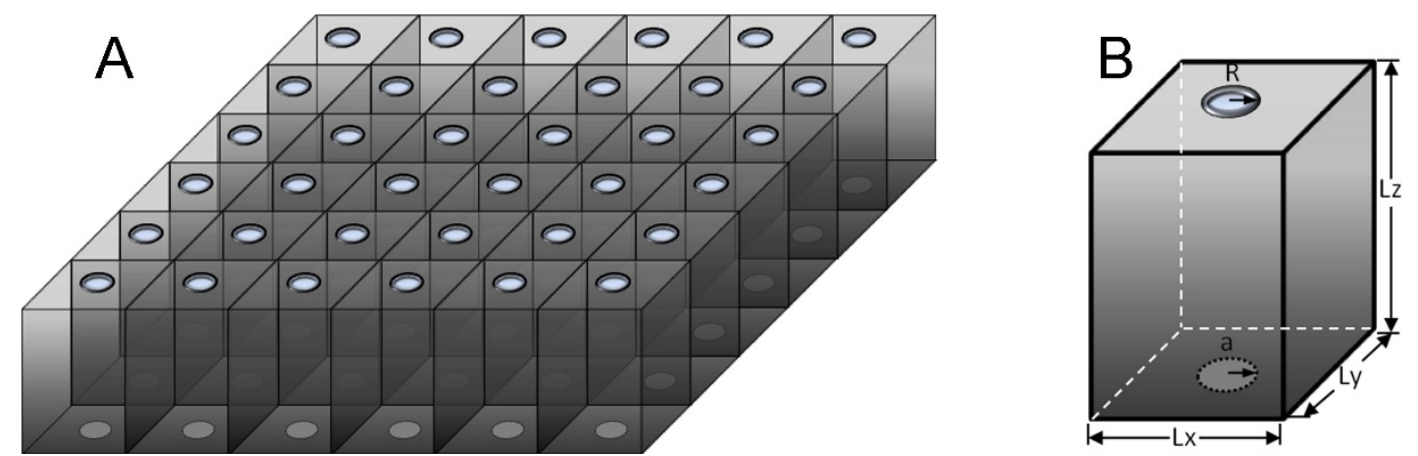

Figure 3 Our model for both a neuromuscular junction and a dyadic cleft. Panel A: The pre-cleft membrane (top) contains a periodic array of disks for the influx of ligands; the post-cleft membrane (bottom) contains a periodic array of absorbing disks representing receptors. Panel B: The dimensions of a unit cell.

the neurotransmitters into the cleft. We model the release of the neurotransmitters as a transient flux, $u(t)$, that is confined to a circular opening with radius $R$. We place the synaptic vesicle at the center of the pre-synaptic face of the unit cell. After diffusing to the postsynaptic membrane, neurotransmitters are absorbed by a circular disk in each unit cell, with radius $a$. We place this "sink" also at the center of the post-synaptic face of the unit cell. The exact shapes and locations of the presynaptic opening and the post-synaptic sink are not essential for the analytical solution of our model. The quantity of interest is the total flux, $J(t)$, through the post-synaptic face of each unit cell.

We place the origin of the Cartesian coordinate system at the center of the pre-synaptic face, with the $z$ axis pointing toward the post-synaptic face. The concentration of neurotransmitters at position $\mathbf{r}$ and at time $t$ is $C(\mathbf{r}, t)$. Within the synaptic junction, it is governed by the diffusion equation,

$$
\frac{\partial C(\mathbf{r}, t)}{\partial t}=D \nabla^{2} C(\mathbf{r}, t)
$$

where $D$ is the diffusion constant. The initial condition is

$$
C(\mathbf{r}, 0)=0
$$

The boundary condition at $z=0$ is

$$
\begin{aligned}
& D \frac{\partial C(\mathbf{r}, t)}{\partial z}=u(t) \quad \text { when } \rho<R \\
& D \frac{\partial C(\mathbf{r}, t)}{\partial z}=0 \quad \text { when } \rho>R
\end{aligned}
$$

where $\rho=\left(x^{2}+y^{2}\right)^{1 / 2}$ is the distance to the $z$ axis. The boundary condition at $z=L_{z}$ is

$$
\begin{aligned}
& C(\mathbf{r}, t)=0 \quad \text { when } \rho<a \\
& D \frac{\partial C(\mathbf{r}, t)}{\partial z}=0 \quad \text { when } \rho>a
\end{aligned}
$$

We solve the problem in Lapace space. For a function $f(t)$ of $t$, we denote the Laplace transform as $\hat{f}(s) \equiv \int_{0}^{\infty} d t e^{-s t} f(t)$. The Laplace transform of Eq. (1), using the initial condition of Eq. (2), is

$$
s \hat{C}(\mathbf{r}, s)=D \nabla^{2} \hat{C}(\mathbf{r}, s)
$$

The solution appropriate for the periodic boundary conditions in the $x$ and $y$ directions has the form

$$
\hat{C}(\mathbf{r}, s)=\sum_{l, m=0}^{\infty}\left[\alpha_{l m}(s) e^{\gamma_{l m}(s) z}+\beta_{l m}(s) e^{-\gamma_{l m}(s) z}\right] \cos \left(2 l \pi x / L_{x}\right) \cos \left(2 m \pi y / L_{\gamma}\right)
$$

where

$$
\gamma_{l m}(s)=\left[s / D+\left(2 l \pi / L_{x}\right)^{2}+\left(2 m \pi y / L_{y}\right)^{2}\right]^{1 / 2}
$$

The coefficients $\alpha_{l m}(s)$ and $\beta_{l m}(s)$ are to be determined from boundary conditions.

To make use of the boundary condition of Eq. (3), we need the 2-dimensional cosine transform of a function in $x$ and $y$ that has value 1 when $\rho<R$ and value 0 when $\rho>R$. The coefficient of the $\cos \left(2 l \pi / L_{x}\right) \cos \left(2 m \pi / L_{y}\right)$ term is

$$
q_{l m}=\frac{4 \varepsilon_{l} \varepsilon_{m}}{L_{x} L_{y}} \iint_{\rho<R} d x d y \cos \left(2 l \pi x / L_{x}\right) \cos \left(2 m \pi y / L_{y}\right)
$$

where $\varepsilon_{0}=1 / 2$ and $\varepsilon_{l}=1$ for $l>0$. For later reference, we note that the function having value 1 when $\rho<a$ 
and value 0 when $\rho>a$ has the following coefficients in its 2-dimensional cosine transform:

$$
p_{l m}=\frac{4 \varepsilon_{l} \varepsilon_{m}}{L_{x} L_{y}} \iint_{\rho<a} d x d y \cos \left(2 l \pi x / L_{x}\right) \cos \left(2 m \pi y / L_{\gamma}\right)
$$

Applying the boundary condition of Eq. (3), we find

$$
D \gamma_{l m}(s)\left[\alpha_{l m}(s)-\beta_{l m}(s)\right]=\hat{u}(s) q_{l m}
$$

For the boundary condition of Eq. (4) at $z=L_{z}$, we use the constant-flux approximation [9]:

$$
\begin{aligned}
& D \frac{\partial \hat{C}(\mathbf{r}, s)}{\partial z}=Q(s) \quad \text { when } \rho<a \\
& D \frac{\partial \hat{C}(\mathbf{r}, s)}{\partial z}=0 \quad \text { when } \rho>a
\end{aligned}
$$

where the quantity $Q(s)$ is to be determined by the condition

$$
<\hat{C}(\mathbf{r}, s)>\equiv\left(\pi a^{2}\right)^{-1} \iint_{\rho<a} d x d \gamma \hat{C}(\mathbf{r}, s)=0
$$

Equation (11) leads to

$$
D \gamma_{l m}(s)\left[\alpha_{l m}(s) e^{\gamma_{l m}(s) L_{z}}-\beta_{l m}(s) e^{-\gamma_{l m}(s) L_{z}}\right]=Q(s) p_{l m}
$$

With Eqs. (10) and (13), we solve for coefficients $\alpha_{l m}$ $(s)$ and $\beta_{l m}(s)$. The results are

$$
\begin{aligned}
& \alpha_{l m} e^{\gamma_{l m}(s) L_{z}}=\frac{1}{D \gamma_{l m}(s)} \frac{-q_{l m} \hat{u}(s)+p_{l m} Q(s) e^{\gamma_{l m}(s) L_{z}}}{e^{\gamma_{l m}(s) L_{z}}-e^{-\gamma_{l m}(s) L_{z}}} \\
& \beta_{l m} e^{-\gamma_{l m}(s) L_{z}}=\frac{1}{D \gamma_{l m}(s)} \frac{-q_{l m} \hat{u}(s)+p_{l m} Q(s) e^{-\gamma_{l m}(s) L_{z}}}{e^{\gamma_{l m}(s) L_{z}}-e^{-\gamma_{l m}(s) L_{z}}}
\end{aligned}
$$

Inserting Eq. (6) in Eq. (12) and using Eqs. (14) and (9), we find $Q(s)$ as

$$
Q(s)=\hat{u}(s) \frac{\sum_{l, m=0}^{\infty} \frac{p_{l m} q_{l m}}{\varepsilon_{l} \varepsilon_{m} \gamma_{l m}(s) \sinh \left[\gamma_{l m}(s) L_{z}\right]}}{\sum_{l, m=0}^{\infty} \frac{p_{l m}{ }^{2} \cosh \left[\gamma_{l m}(s) L_{z}\right]}{\varepsilon_{l} \varepsilon_{m} \gamma_{l m}(s) \sinh \left[\gamma_{l m}(s) L_{z}\right]}}
$$

Finally the total flux through the sink on the postsynaptic face is

$$
\hat{J}(s)=\pi a^{2} Q(s)
$$

The flux accumulated over all times,

$$
I=\int_{0}^{\infty} d t J(t)
$$

is of interest. In our model,

$$
I=\hat{J}(0)=\pi R^{2} \hat{u}(0)
$$

which is independent of $a$. Equation (17b) is simply a consequence of ligand conservation, i.e., the total number of ligands released from the synaptic vesicle is the same as the total number of ligands absorbed by the receptors.

The analytical solution derived above can be implemented on any function $u(t)$ modeling neurotransmitter release from the synaptic vesicle. We focused on an exponentially decaying function:

$$
u(t)=e^{-t / t_{0}}
$$

Its Laplace transform is

$$
\hat{u}(s)=\frac{1}{s+1 / t_{0}}
$$

Our model then contains two parameters related to time: $D$ and $t_{0}$. For two sets of these parameters, e.g., $D_{1}$ and $t_{01}$ in one and $D_{2}$ and $t_{02}$ in the other, it can be shown that the corresponding response functions satisfy

$$
J_{1}\left(D_{2} t\right)=J_{2}\left(D_{1} t\right) \quad \text { when } D_{1} t_{01}=D_{2} t_{02}
$$

We now briefly describe the details of our implementation of the analytical solution. To calculate the $q_{l m}$ and $p_{l m}$ coefficients of Eqs. (8) and (9), we first carried out the integration over $y$ analytically. The remaining integration over $x$ was done numerically using the Gauss-Legendre quadrature with two points. The summations over $l$ and $m$ in Eq. (15) were truncated at $l=$ $m=40$. The Laplace transform of $\hat{J}(s)$ was inverted by the Stehfest algorithm [10]. A Fortran90 code for the implementation is available upon request.

\section{Results}

We now present some illustrative results. The parameters of our model are as follows: $L_{x}=L_{y}=500 \mathrm{~nm}$; $L_{z}=50 \mathrm{~nm} ; R=20 \mathrm{~nm} ; a$ varied from 2.5 to $40 \mathrm{~nm} ; t_{0}$ varied from 1 to $10 \mathrm{~ms}$; and $D$ varied in $(0.4-4) \times 10^{5}$ $\mathrm{nm}^{2} / \mathrm{ms}$.

Our single absorbing disk is used to model ligand binding to multiple receptors. Increasing $a$ thus mimics an increasing number, $N_{\text {rec }}$, of receptors per unit cell. 
We expect $a$ to increase linearly with increasing $N_{\text {rec }}$ when $N_{\text {rec }}$ is small; the increase in $a$ then slows down at higher $N_{\text {rec }}$ (see Discussion). Figure 4 displays the dependence of the response function $J(t)$ on $a$ (and hence $N_{\text {rec }}$ ). As a (i.e., $N_{\text {rec }}$ ) increases, $J(t)$ more and more quickly reaches a higher and higher maximum and decays faster and faster. This is the expected behavior. Moreover, according to Eqs. (17b) and (18b), the areas under the curves for different $a$ values are all equal to $\pi R^{2} t_{0}$. Note also that all our $J(t)$ curves have the familiar shape seen in previously numerical studies [1-7].

Figure 5 shows the change in the response function when the speed at which the neurotransmitters are released into the synaptic cleft is varied. To make a fair comparison, $J(t)$ is scaled by $t_{0}$ so that, effectively, the total number of neurotransmitters entering the synaptic cleft is fixed. It is clear that, as $t_{0}$ increases (i.e., as the speed of neurotransmitter release decreases), the response function rises and decays more slowly. This behavior has previously been specifically modeled by Stiles et al. [1].

There is some uncertainty on the diffusion constant of acetylcholine in neuromuscular junctions. In previous models [1-6], the value of $D$ ranged from $(0.25-6) \times 10^{5}$ $\mathrm{nm}^{2} / \mathrm{ms}$. In Figure 6 we display the change in the response function when $D$ is varied from $(0.4-4) \times 10^{5}$ $\mathrm{nm}^{2} / \mathrm{ms}$. As expected, when neurotransmitter diffusion slows down, the response function is also delayed. In our model, decreasing $D$ has the same effect on the response function as increasing $t_{0}$ [see Eq. (19)].

\section{Discussion and Conclusion}

We have presented an analytical solution to a model for the diffusion and reaction of acetylcholine in a

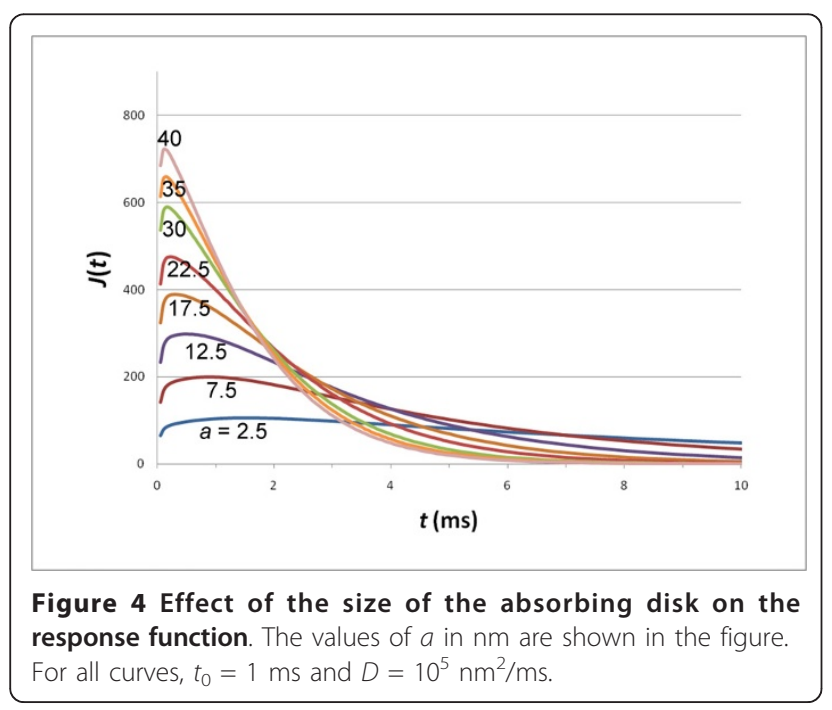

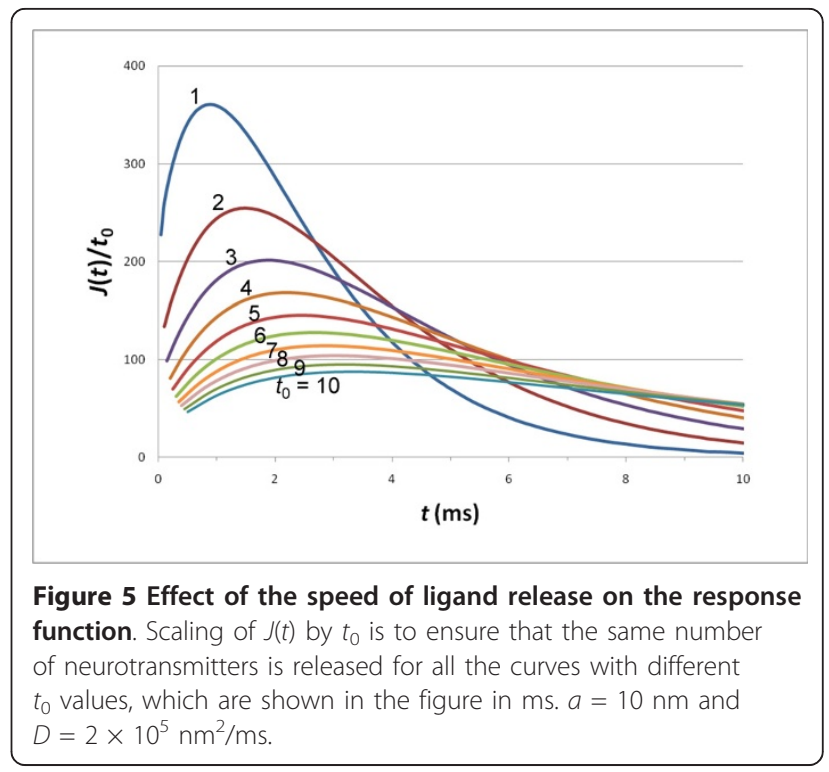

neuromuscular junction. The model also applies to the diffusion and binding of $\mathrm{Ca}^{2+}$ in a dyadic cleft. Our results are qualitatively similar to those obtained previously from models solved numerically [1-7].

Perhaps the greatest value of our analytical solution is that it provides a benchmark for testing numerical methods. Diffusion and reaction of ligands in intercellular and intracellular spaces have been modeled either on a particle description or a concentration description. The former type of models have been solved by Monte Carlo simulations [1,7], while the latter type of models have been solved by either finite-difference [2,6] or finite-element [3-5] methods. The two types of models have been shown to give equivalent results [8]. The level of realism of our model approaches those of the models solved numerically;

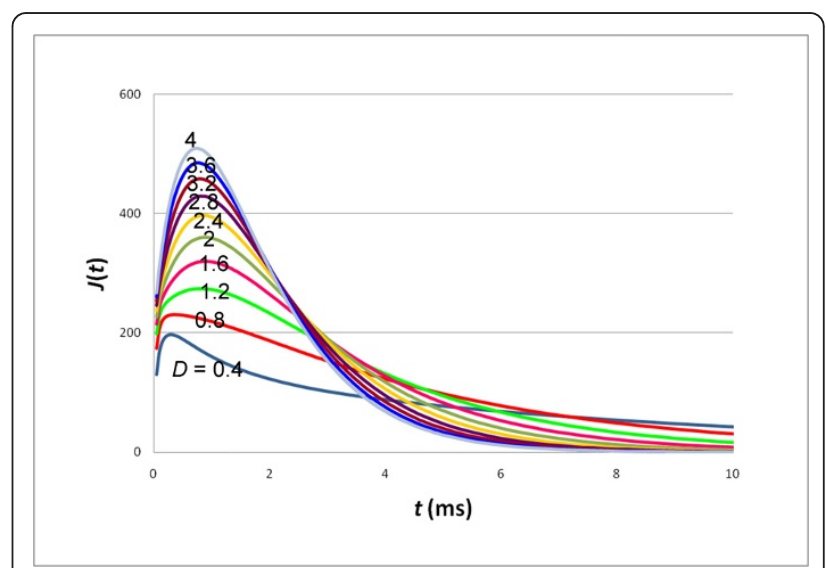

Figure 6 Effect of the ligand diffusion constant on the response function. The values of $D$ in $10^{5} \mathrm{~nm}^{2} / \mathrm{ms}$ are shown in the figure. For all curves, $a=10 \mathrm{~nm}$ and $t_{0}=1 \mathrm{~ms}$. 
hence our analytical solution will be able to serve as a good benchmark for the numerical methods.

Our model has room for increasing the level of realism and still allows for analytical solution. For example, we modeled ligand binding to receptors as absorbing. The binding can be modeled as partially absorbing if binding does not occur at every ligand-receptor encounter. The partial absorption condition takes the form

$$
D \frac{\partial C(\mathbf{r}, t)}{\partial z}=\kappa C(\mathbf{r}, t) \quad \text { when } \rho<a
$$

where the reactivity $\kappa$ controls the degree of partial absorption. When $\kappa=0$, the region $\rho<a$ becomes reflecting and binding cannot occur. When $\kappa \rightarrow \infty$, the region $\rho<a$ becomes fully absorbing and Eq. (20) reduces to the absorbing condition of Eq. (4a). Note that the bimolecular rate constant $k$ for binding to the partially absorbing disk is given by [11]

$$
\frac{1}{k}=\frac{1}{4 D a}+\frac{1}{\pi \kappa a^{2}}
$$

One can thus parameterize $a$ and $\kappa$ by matching $k$ with experimental data for the ligand-receptor binding rate constant.

Another simplification of our model is that a single absorbing disk is used to represent the receptors. We accounted for the presence of multiple receptors per unit cell by increasing the radius $a$ of this disk. One can identify $a$ by requiring that the bimolecular rate constant $k$ calculated for the single absorbing disk is the same as that for the $N_{\text {rec }}$ receptors. For the single absorbing disk we have $k=4 D a$ [see Eq. (21)]. If each of the $N_{\text {rec }}$ receptors is modeled as an absorbing disk with a small radius $a_{0}$, then $k \approx 4 N_{\text {rec }} D a_{0}$ when $N_{\text {rec }}$ is small [12]. Therefore $a \approx N_{\text {rec }} a_{0}$ for small $N_{\text {rec }}$. As $N_{\text {rec }}$ increases, the rate constant $k$ for the multiple receptors and correspondingly the radius $a$ of the equivalent disk also increase, but the increase slows down as $N_{\text {rec }}$ becomes large $[12,13]$. Instead of using a single equivalent absorbing disk, analytical solution is actually still permitted when multiple receptors, each represented by a (partially) absorbing small disk, are present at arbitrary positions on the post-synaptic face. An alternative way to model the presence of multiple receptors is to assume that the whole post-synaptic face is partially absorbing, with an reactivity given by $[12,13]$

$$
\mathrm{K}=\frac{N_{\mathrm{rec}} k_{0}}{S\left(1-f_{\mathrm{rec}}\right)}
$$

where $S=L_{x} L_{y}$ is the area of the post-synaptic face, $f_{\text {rec }}=N_{\text {rec }} \pi a_{0}^{2} / S$ is the fraction of the post-synaptic face that is covered by the receptors, and $k_{0}$ is given by

$$
\frac{1}{k_{0}}=\frac{1}{4 D a_{0}}+\frac{1}{\pi \kappa a_{0}^{2}\left(1-f_{\mathrm{rec}}\right)}
$$

We have modeled ligand-receptor binding as irreversible. This is somewhat justified for modeling the neuromuscular junction, in which acetylcholinesterase can break down acetylcholine molecules newly released from the receptors. No such mechanism is present for $\mathrm{Ca}^{2+}$ in the dyadic cleft. Reversible binding can be treated by appropriate boundary conditions [14] on the post-synaptic face. Another important detail is that both acetylcholine and ryanodine receptors have multiple binding sites for their ligands so that there are multiple ligand-occupation states for the receptors. Again, these can be treated by appropriate boundary conditions.

The geometries of some of the models previously solved numerically are more sophisticated than that of our model. In particular, secondary folds of the neuromuscular junction has been included in some of the previous models [1,3-5]. A formalism for treating ligand binding to a site buried in a narrow tunnel has been developed [15] and may be adopted for treating the narrow secondary folds in the neuromuscular junction. However, analytical solution requires idealized geometries; the kind of realistic geometries drawn from electron microscopy that can be handled by a finite-element method $[4,5]$ is beyond the reach of analytical solution. Nevertheless, with all the new ingredients outlined above, analytical solution will potentially provide a new avenue for modeling biochemical transport.

\section{Acknowledgements}

This work was supported in part by Grant GM58187 from the National Institutes of Health.

\section{Authors' contributions}

$J L B$ implemented the analytical solution, did the calculations, and prepared the figures. HXZ derived the analytical solution and wrote the paper. Both authors read and approved the final manuscript.

Received: 9 February 2011 Accepted: 2 March 2011

Published: 2 March 2011

\section{References}

1. Stiles JR, Van Helden D, Bartol TM Jr, Salpeter EE, Salpeter MM: Miniature endplate current rise times less than 100 microseconds from improved dual recordings can be modeled with passive acetylcholine diffusion from a synaptic vesicle. Proc Natl Acad Sci USA 1996, 93:5747-5752.

2. Naka T, Shiba K, Sakamoto N: A two-dimensional compartment model for the reaction-diffusion system of acetylcholine in the synaptic cleft at the neuromuscular junction. Biosystems 1997, 41:17-27.

3. Smart JL, McCammon JA: Analysis of Synaptic Transmission in the Neuromuscular Junction Using a Continuum Finite Element Model. Biophysical journal 1998, 75:1679-1688.

4. Tai K, Bond SD, MacMillan HR, Baker NA, Holst MJ, McCammon JA: Finite element simulations of acetylcholine diffusion in neuromuscular junctions. Biophys J 2003, 84:2234-2241. 
5. Cheng Y, Suen JK, Radic Z, Bond SD, Holst MJ, McCammon JA: Continuum simulations of acetylcholine diffusion with reaction-determined boundaries in neuromuscular junction models. Biophys Chem 2007, 127:129-139.

6. Khaliq A, Jenkins F, DeCoster M, Dai W: A new 3 D mass diffusion-reaction model in the neuromuscular junction. Journal of Computational Neuroscience 2010, 1-17.

7. Koh X, Srinivasan B, Ching HS, Levchenko A: A 3 D Monte Carlo analysis of the role of dyadic space geometry in spark generation. Biophys J 2006, 90:1999-2014.

8. Hake J, Lines GT: Stochastic binding of $\mathrm{Ca} 2+$ ions in the dyadic cleft; continuous versus random walk description of diffusion. Biophys J 2008, 94:4184-4201.

9. Shoup D, Lipari G, Szabo A: Diffusion-controlled bimolecular reaction rates. The effect of rotational diffusion and orientation constraints. Biophysical Journal 1981, 36:697-714.

10. Stehfest H: Algorithm 368: Numerical inversion of Laplace transform. Communication of the ACM 1970, 13:47-49.

11. Zhou H-X, Szabo A: Theory and simulation of the time-dependent rate coefficients of diffusion-influenced reactions. Biophysical Journal 1996, 71:2440-2457.

12. Shoup D, Szabo A: Role of diffusion in ligand binding to macromolecules and cell-bound receptors. Biophysical Journal 1982, 40:33-39.

13. Zwanzig R: Diffusion-controlled ligand binding to spheres partially covered by receptors: an effective medium treatment. Proc Natl Acad Sci USA 1990, 87:5856-5857.

14. Gopich IV, Szabo A: Kinetics of reversible diffusion influenced reactions: the self-consistent relaxation time approximation. Journal of Chemical Physics 2002, 117:507-517.

15. Zhou H-X: Theory of the diffusion-influenced substrate binding rate to a buried and gated active site. Journal of Chemical Physics 1998, 108:8146-8154.

doi:10.1186/2046-1682-4-5

Cite this article as: Barreda and Zhou: A solvable model for the diffusion and reaction of neurotransmitters in a synaptic junction. $B M C$ Biophysics 2011 4:5.

\section{Submit your next manuscript to BioMed Central and take full advantage of:}

- Convenient online submission

- Thorough peer review

- No space constraints or color figure charges

- Immediate publication on acceptance

- Inclusion in PubMed, CAS, Scopus and Google Scholar

- Research which is freely available for redistribution

Submit your manuscript at www.biomedcentral.com/submit 